\title{
Application of a multi-scale form of Terzaghi's effective stress principle for unsaturated expansive clays to simulate hydro-mechanical behavior during hydration
}

\author{
Julia Mainka ${ }^{1, a}$, Marcio A. Murad ${ }^{2}$, Van Duy $\operatorname{Tran}^{1}$, Tien Dung LE ${ }^{1}$ and Christian Moyne ${ }^{1}$ \\ ${ }^{1}$ LEMTA - Université de Lorraine - CNRS - UMR 7563, Vandœuvre-lès-Nancy, France \\ ${ }^{2}$ LNCC, avenida Getúlio Vargas 333, 25651-075 Petrópolis, RJ, Brasil
}

\begin{abstract}
Our recently developed multi-scale form of Terzaghi's effective stress principle for unsaturated swelling clays that was rigorously derived by periodic homogenization starting from micro- and nano-mechanical analyses is applied to numerically simulate one-dimensional swelling pressure tests of compacted bentonites during hydration. The total macroscopic stress captures the coupling between disjoining forces at the nanoscopic scale of clay platelets and capillary effects at the microscopic scale of clay aggregates over the entire water content range. The numerical results allow to draw conclusions on the water transfer mechanism between inter- and intra-aggregate pores during hydration and consequently on the evolution of the external swelling pressure resulting from the competition between capillary and disjoining forces. In addition, such application highlights the abilities and the limits of the electrical double-layer theory to compute the disjoining pressure in the nano-pores. For large platelet distances, in the range of osmotic swelling, the nature of the disjoining pressure is electro-chemical and can be computed from PoissonBoltzmann theory. Conversely, at small distances, in the crystalline swelling, a solvation component has to be added to account for the molecular nature of the solvent. As a first improvement of the nano-scale description the solvent is treated as a hard sphere fluid using Density Functional Theory.
\end{abstract}

\section{Introduction}

A variety of experimental $[1,2]$ and modelling efforts [3-5] have been undertaken to analyze the hydromechanical behaviour of expansive clays during water infiltration by means of one-dimensional swelling pressure tests. The basic results are: (i) The swelling pressure increases during hydration and reaches values of some MPa at the fully saturated state. The final value is basically linked to dry density [2]. (ii) After an initial fast increase, the swelling pressure exhibits a temporary decrease followed by a new increase until reaching a stationary value being of the same amplitude as the first maximum $[1,2,6,7]$. This behaviour is associated with the collapse of the macroscopic solid structure when reaching the loading-collapse (LC) line [2].

It is now well recognized that the microstructure plays a crucial role in the evolution of the overall swelling pressure. In order to enhance the understanding of the processes underlying the experimental observations, several modelling efforts were undertaken. Among them we may highlight double-porosity models [3-5,8-10]. Recent works include the hydro-mechanical behaviour of non-expansive [10] and expansive unsaturated clays [2$5,9]$. When accounting for elasto-plastic deformation, the evolution of the swelling pressure could be reproduced including its temporary drop [2], as well as the

\footnotetext{
a Corresponding author: julia.mainka@univ-lorraine.fr
}

reorganization of the macrostructure [3-5,10]. All models are able to link the mechanical behaviour to capillary pressure drop in the porous space. On the other hand, to the best of our knowledge they are all based on an empirical approach to account for micro- and nanoscopic contributions to the overall swelling pressure.

This work tries to fill this gap. Our double-porosity model that was rigorously derived by micro-mechanical analyses [9] is applied to numerically simulate the swelling pressure evolution during hydration at fixed volume. Starting from a formulation of the mechanical equilibrium at the nano-scale of the electrical-double layer (EDL) and the micro-scale where capillary effects take place, the coupling between these two effects is incorporated over the entire saturation range. As will be shown, the model provides furthermore insight on the water transfer mechanism from the micro- to the nanoporous space and the linked evolution of the disjoining pressure during hydration. In addition, we show the limits of the electrical-double layer theory when computing the disjoining pressure at low hydration levels. Note that only elastic deformation is considered at the micro- and macro-scale. Ongoing work is in progress to incorporate elasto-plasticity in the multi-scale approach. 


\section{Theory}

\subsection{Isotropic form of the multi-scale model}

The detailed derivation of our multi-scale Terzaghi's effective stress principle and all model equations are presented in a former paper [9]. In the following, we present directly the final macroscopic model equations in an isotropic medium approximation recalling the principle ideas underlying their derivation.

The model is based on a given representation of the microstructure of expansive clays which is characterized by two porosity levels (referred to nano- and micro-pores tied up their size [11]) and three disparate length scales as shown on Figure 1.

- At the nano-scale the medium is composed of incompressible linear-elastic clay platelets of constant surface charge, $\sigma<0$. The platelets are separated by a nano-porous network saturated by a binary electrolyte solution (considered as continuous in classical EDL approach) with monovalent completely dissociated ions considered as point charges.

- At the intermediate micro-scale the platelets form assemblies of swollen clay aggregates (or particles) constituting the solid phase at that length scale. The particles are separated by a micro-porous network (free of EDL effects regarding their size) which is filled by a mixture of bulk water and air.

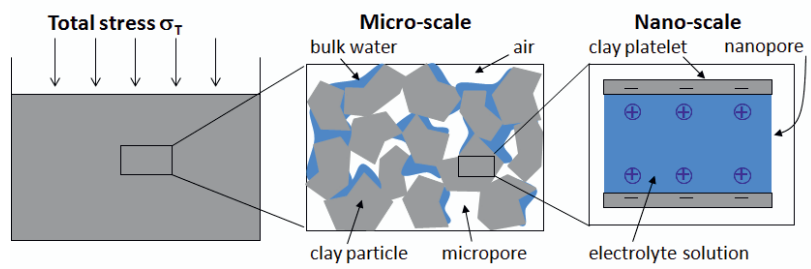

Figure 1. Multi-scale structure of swelling clays.

Starting from the formulation of the mechanical equilibrium at the local scales, the overall total stress is obtained using periodic homogenization [12]. Supposing no preferential orientation of the local clay fabric, all stresses reduce to pressures and the total stress is given by:

$$
\boldsymbol{\sigma}_{T}=-P_{e x t} \mathbf{I}=\sigma_{E} \mathbf{I}-P_{f}^{e f f} \mathbf{I}-\Pi_{e f f} \mathbf{I},
$$

with $\mathrm{P}_{\text {ext }}$ the external total pressure and $\mathbf{I}$ the identity tensor. From the above equation we may note that $\mathrm{P}_{\text {ext }}$ is given by the sum of three terms (i) a net contact stress $\sigma_{\mathrm{E}}$, (ii) an effective fluid pressure $\mathrm{P}_{\mathrm{f}}^{\text {eff }}$ accounting for capillary effects arising from the micropores and (iii) an effective disjoining pressure $\Pi_{\text {eff }}$ strongly tied up with the action of the electrolyte solution on the clay platelets at the nano-scale. The elastic contact stress $\sigma_{E}=\operatorname{tr}\left(\boldsymbol{\sigma}_{E}\right) / 3$ is linked to the volumetric macroscopic strain $\varepsilon=\operatorname{tr}(\varepsilon)$ by Hooke's law $\sigma_{E}=\tilde{K} \varepsilon$. The macroscopic bulk modulus $\tilde{K}$ is expressed as a function of the micro-porosity $\mathrm{n}_{\mathrm{f}}$, the microscopic bulk modulus of the clay particles $\mathrm{K}_{\mathrm{s}}$ and the microscopic Poisson ratio $v_{\mathrm{s}}$ using self-consistent medium approximation according to [12]. The equivalent fluid pressure reads as

$$
P_{f}^{e f f}=P_{a}-\chi^{e f f} P_{c},
$$

with $P_{a}$ the air pressure and $P_{c}$ the capillary pressure defined by the negative water pressure $\mathrm{P}_{\mathrm{c}}=\mathrm{P}_{\mathrm{a}}-\mathrm{P}_{\mathrm{w}}$. $\mathrm{P}_{\mathrm{c}}$ can be linked to the overall water saturation $S_{w}$ by a Van Genuchten type law. $\chi^{\text {eff }}$ is the volumetric component the effective Bishop type tensor which is linked to clay fabric properties and saturation in the micropores according to

$$
\chi^{\text {eff }}=1-(1-\chi) \alpha,
$$

where $\chi(0 \leq \chi \leq 1)$ is the microscopic Bishop parameter which can be expressed as a function of saturation, for example according to [13]. In addition, $\alpha$ is the macroscopic Biot coefficient defined in a classical way by $\alpha=1-\tilde{K} / K_{s}$ represented in the form

$$
\alpha=1-\frac{2\left(1-n_{f}\right)\left(1-2 v_{s}\right)}{\left(1+v_{s}\right) n_{f}+2\left(1-2 v_{s}\right)} .
$$

The macroscopic result of the nanoscopic disjoining pressure $\Pi_{d}$ is finally given by

$$
\Pi^{\text {eff }}=(1-\alpha) \Pi_{d} .
$$

We may observe that $\alpha$ plays the role of a scaling factor accounting for the transmissibility of disjoining forces between adjacent clay particles. $\Pi_{d}$ is generally expressed as a function of ion concentration $\mathrm{c}_{\mathrm{b}}$ and interplatelet distance $2 \mathrm{H}$ using Poisson-Boltzmann (PB) equation [8]

$$
\Pi_{d}=2 c_{b} R T\left(\cosh \frac{F \varphi_{0}}{R T}-1\right),
$$

with $\mathrm{R}$ the perfect gas constant, $\mathrm{T}$ the absolute temperature, $F$ the Faraday constant and $\varphi_{0}=\varphi_{0}\left(\mathrm{c}_{\mathrm{b}}, \mathrm{H}\right)$ the electric potential at the midplane between two adjacent platelets.

This is the classical approach for osmotic swelling where disjoining forces are driven by EDL effects. For small inter-platelet distances however, in the crystalline swelling regime, the molecular nature of solvent and ions is not more negligible and the disjoining pressure should be dominated by particle size effects [14] which could be included using Density Functional Theory (DFT) [15]. DFT allows to obtain particle density distribution $\rho(z)$ (with $\mathrm{z}$ the distance to the platelet surface for isotropic fluids) at equilibrium as a function of the excess free energy functional $F^{\mathrm{ex}}[\rho]$ by minimizing the appropriate thermodynamic potential, here the grand canonical potential.

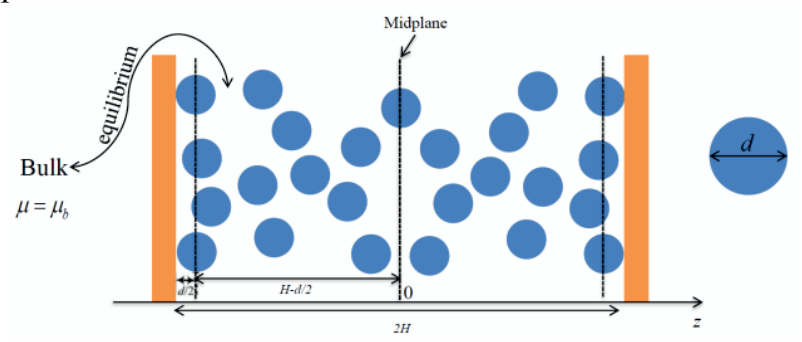

Figure 2. Improved nano-scale description considering the electrolyte solution as a hard sphere fluid confined between two planar hard walls.

As a first improvement of the nano-scale description, we consider in the present work the molecular nature of the solvent only (ion size and concentration are negligible compared to those of water). By considering the electrolyte solution as a simple hard sphere (HS) fluid of 
diameter d confined between two planar hard walls (Figure 2), $F^{\mathrm{ex}}[\rho]$ is expressed by Fundamental Measure Theory (FMT) according to [16]. The disjoining pressure being defined as the force acting on the walls to keep them at a fixed distance $2 \mathrm{H}$, it can be expressed using contact theorem which tells that, at mechanical equilibrium, $\Pi_{d}$ can be computed from the difference between the pressure the fluid acts on the wall $\mathrm{P}$ and the bulk pressure $\mathrm{P}_{\mathrm{b}}$ :

$$
\begin{aligned}
\Pi_{d}(2 H) & =P(2 H)-P_{b}=k_{B} T\left[\rho_{\text {contact }}(2 H)-\rho_{C S}\left(\rho_{b}\right)\right] \\
& =k_{B} T\left[\rho(z=d / 2,2 H)-\rho_{C S}\left(\rho_{b}\right)\right]
\end{aligned}
$$

with $\mathrm{k}_{\mathrm{B}}$ the Boltzmann constant. The density distribution of the bulk fluid $\rho_{\mathrm{CS}}$ is expressed in terms of the bulk density $\rho_{\mathrm{b}}$ by Carnahan-Starling equation [17].

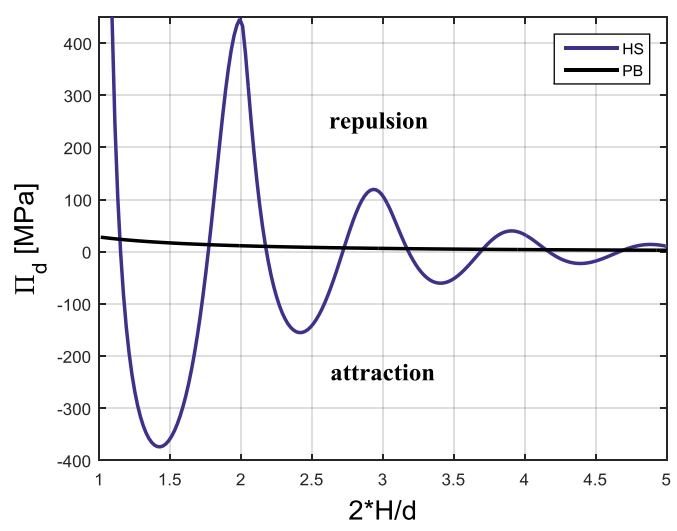

Figure 3. Disjoining pressure profiles of a PB fluid $\left(c_{b}=10^{-4}\right.$ mol/l) and a HS fluid $\left(\rho_{b} d^{3}=0.73\right)$ as a function of normalized inter platelet distance $2 \mathrm{H} / \mathrm{d}\left(\mathrm{d}_{\mathrm{H} 20}=2.8 \AA\right)$.

Figure 3 compares the profiles of the disjoining pressure obtained by PB theory $\left(\mathrm{c}_{\mathrm{b}}=10^{-4} \mathrm{~mol} / \mathrm{l}\right)$ and for a HS fluid as a function of normalized inter platelet distance $2 \mathrm{H} / \mathrm{d}$. For the HS fluid, the water molecule diameter and normalized bulk concentration are set to $\mathrm{d}_{\mathrm{H} 20}=2.8 \AA$ and $\rho_{\mathrm{b}} \mathrm{d}^{3}=0.73$ (corresponding to a mass density $\rho_{\mathrm{H} 20}=10^{3} \mathrm{~kg} / \mathrm{m}^{3}$ ). The HS fluid pressure allows to account for attraction $\left(\Pi_{d}<0\right)$ and repulsion $\left(\Pi_{d}>0\right)$, while $\mathrm{PB}$ is a purely repulsive decreasing function of the platelet distance. According to the expectations [14], the oscillatory profile of the HS fluid exhibits maxima at entire multiples of $\mathrm{d}$ whose amplitude decreases rapidly with the platelet distance. Note that only negative slope parts represent equilibrium positions. In addition, the HS law reaches somewhat higher values of the disjoining pressure at small distances than PB theory.

The coupling of the different physical phenomena is made through the porosities at the micro- and nano-scale $\phi$ being defined as the respective pore volume over the total volume. Assuming a stratified local arrangement, the nano-porosity is expressed by the inter-platelet distance according to $\phi=H /\left(H+H_{s}\right)$, with $2 \mathrm{H}_{\mathrm{S}}$ the platelet thickness. For an isotropic homogeneous distribution of the clusters, the two porosities are linked by a scalar version of the overall solid mass balance

$$
\left(1-n_{f}\right)(1-\phi)=\left(1-\bar{n}_{f}\right)(1-\bar{\phi}) \exp (-\varepsilon)
$$

where the overbars refer to a stress free reference state which can be chosen arbitrarily. Similarly, using selfconsistent approach, the micro-porosity is given by an integral form of the fluid mass balance

$$
n_{f}=\bar{n}_{f}+\frac{n_{f}\left(1-n_{f}\right)}{\frac{2\left(1-2 v_{s}\right)}{1+v_{s}}+n_{f}}\left(\varepsilon+\frac{(1-\chi) P_{c}}{K_{s}}-\frac{\Pi_{d}}{K_{s}}\right) \text {. }
$$

\subsection{Application to an oedometric swelling test}

In the following, we present the governing equations that underlie the computation of the swelling pressure $\mathrm{P}_{\mathrm{ext}}$ at different stages of wetting, for the dry and fully saturated states and along with intermediate saturations. The numerical application refers to an experimental swelling pressure test on FoCa clay 50/50 weight percent bentonite powder/pellets mixture performed at the CIEMAT and presented in [1,2]. The clay sample we refer to is $M G R 7$ with a dry density $\rho_{\text {dry }}=1.45 \mathrm{~g} / \mathrm{cm}^{3}$ which corresponds to the ratio of the mass of the solid phase (clay cluster) at the dry state $m_{c}{ }^{i}$ over the total volume $V_{T}$. The cylindrical sample $(100 \mathrm{~mm}$ in diameter and $50 \mathrm{~m}$ in height) was hydrated from the bottom of an oedometric cell. The swelling pressure applied to the cell to prevent deformation during water intake was recorded for mean water contents $w$ varying from $w_{\mathrm{i}}=0.8$ to $w_{\mathrm{f}}=0.33$.

\subsubsection{Initial "dry state"}

Initially, we assume all water to be confined in the nanopores so that the micropores are only filled by air implying that $\chi^{i}=0$. The gas/liquid interface should thus be at clay cluster surface (cf. Figure 4). This hypothesis agrees with a rough estimation of the pore diameter linked to the initial capillary pressure of about $200 \mathrm{MPa}$ using Laplace equation $2 R=4 \gamma / P_{c} \approx 2 \mathrm{~nm} \quad$ (with water/air surface tension $\left.\gamma(298 \mathrm{~K})=72 \times 10^{-3} \mathrm{~N} . \mathrm{m}\right)$ which is clearly superior to the nanopores.

The initial state represents the reference state for this application, so that $\bar{n}_{f}=n_{f}^{i}$ and $\bar{\phi}=\phi^{i}$. As sample deformation is prevented during water intake no overall elastic stress appears with respect to the initial state and $\sigma_{\mathrm{E}}=0$. Initially there is no pressure applied to the cell $\mathrm{P}_{\text {ext }}{ }^{\mathrm{i}}=0$ and it follows from (1) in combination with (2), (3) and (5) that all capillary effects are compensated by the disjoining pressure $\Pi_{d}^{i}=P_{c}^{i}=200 M P a$.

As $w_{\mathrm{i}}=0.8$, it is reasonable to assume crystalline swelling [18] so that $\Pi_{d}{ }^{i}$ is computed according to the HS fluid law in (7). The inter platelet distance estimated with this law, $2 \mathrm{H}^{\mathrm{i}}=3.12 \AA$, corresponds to $1-2$ water layers which is consistent with crystalline swelling. Assuming a platelet thickness $2 \mathrm{H}_{\mathrm{s}}=1 \mathrm{~nm}$, the corresponding nanoporosity is $\phi^{\mathrm{i}}=\mathrm{H}^{\mathrm{i}} /\left(\mathrm{H}^{\mathrm{i}}+\mathrm{H}_{\mathrm{s}}\right)=0.24$.

It is important to note that using PB theory, the disjoining pressure is not able to compensate the initial capillary pressure (cf. Figure 3). Even if the approximation of the electrolyte solution by a simple HS fluid requires further improvement including ionic and 
dipolar interactions [19], the proposed pore treatment presents an improved nano-scale description at low hydration where the molecular nature dominates over EDL effects and where small pore diameter deviations imply important disjoining pressure variations.

For the estimation of the micro-porosity we introduce the platelet density $\rho_{\mathrm{ss}}=\mathrm{m}_{\mathrm{sc}} / \mathrm{V}_{\mathrm{sc}}=2670 \mathrm{~kg} / \mathrm{m}^{3}$ [20], with $\mathrm{m}_{\mathrm{sc}}$ and $\mathrm{V}_{\mathrm{sc}}$ the mass and the volume of the solid phase in a clay cluster. All water being located in the nanopores the water content is $w^{\mathrm{i}}=\mathrm{m}_{\mathrm{H} 20, \mathrm{c}}^{\mathrm{i}} / \mathrm{m}_{\mathrm{sc}}$ and it follows

$$
\frac{\rho_{d r y}}{\rho_{s s}\left(1-\phi^{i}\right)}=\frac{m_{c}^{i}}{V_{T}} \frac{V_{s c}}{m_{s c}} \frac{V_{c}^{i}}{V_{s c}}=\frac{V_{c}^{i}}{V_{T}} \frac{m_{s c}+m_{H 20, c}^{i}}{m_{s c}}=\left(1-n_{f}^{i}\right)\left(1+w^{i}\right)
$$

From this relation we obtain $\mathrm{n}_{\mathrm{f}}^{\mathrm{i}}=0.39$ which is quite similar to the experimental value given in [2].

\subsubsection{Final "saturated state"}

At full saturation the capillary pressure vanishes and from (2) it follows that the fluid pressure contribution becomes negligible $\mathrm{P}_{\mathrm{f}}^{\text {eff, } \mathrm{f}}=\mathrm{P}_{\mathrm{a}} \approx 0$. From the mechanical equilibrium (1) it can be concluded that the swelling pressure corresponds to the effective disjoining pressure

$$
P_{e x t}^{f}=\Pi_{e f f}^{f}=\left(1-\alpha^{f}\right) \Pi_{d}^{f},
$$

where $\Pi_{d}{ }^{f}$ is a function of $H$ (or $\phi$ ) expressed by classical PB theory according to (6). This hypothesis seems reasonable as disjoining stresses may originate from osmotic swelling at full saturation.

\subsubsection{Intermediate saturations}

At intermediate saturations we chose a representation of the overall medium by a division into two parts (cf. Figure 4): a saturated part of height $x+\Delta$ at the bottom and a part of height L-X- $\Delta$ (L being the sample height) at the top being at the initial state. $\Delta$ is the local macroscopic deformation due to the difference in water content between both parts. This corresponds not necessarily to the real configuration, as no information is given about the advancement of the water front during infiltration. The advantage of this approximation is that it does not require defining microscopic saturation and capillary pressure which can differ from their macroscopic values.
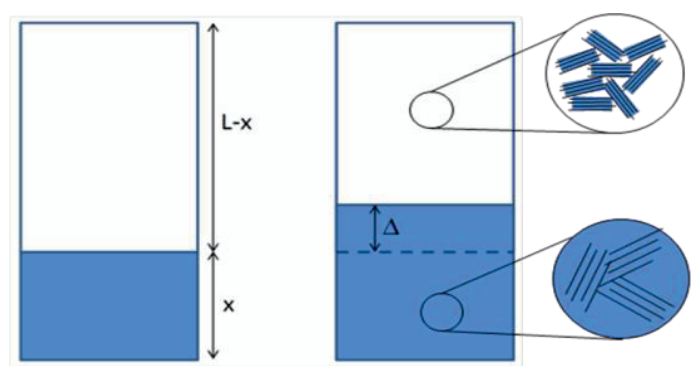

Figure 4. Representation of the sample at intermediate saturation: the bottom is fully saturated and the top at the initial state. The overall saturation is obtained by the volume ratio of the two parts. On the left: configuration without deformation; on the right: with deformation $\Delta$ due to different water contents.

For a constant sample diameter, the volumetric fractions of the two parts are given by the ratio of their respective height to the sample height, i.e. $\theta_{\mathrm{b}}=\mathrm{x}+\Delta / \mathrm{L}$ and $\theta_{\mathrm{t}}=1-\theta_{\mathrm{b}}$. Volume fraction is linked to the overall saturation by

$$
S_{w}=\theta_{b}+\left(1-\theta_{b}\right) S_{w}^{i},
$$

with $\mathrm{S}_{\mathrm{w}}{ }^{\mathrm{i}}=w^{\mathrm{i}} / w^{\mathrm{f}}=0.26$. Even if overall sample deformation is prevented, local vertical deformation occurs in both parts $\varepsilon=\{0,0, \varepsilon\}$ which are expressed as

$$
\varepsilon_{b}=\frac{\Delta}{x} \text { and } \varepsilon_{t}=-\frac{\Delta}{L-x},
$$

where the coupling with the saturation is made by (12). The corresponding oedometric elastic pressures are $\sigma_{E}^{b / t}=\tilde{M}^{b / t} \varepsilon^{b / t} \quad$ with $\quad \tilde{M}^{b / t}=\tilde{K}^{b / t}+4 / 3 \mu^{b / t} \quad$ the oedometric bulk moduli. Using (4) in combination with the relation $\mu=3(1-2 v) /[2(1+v)] \tilde{K}$ for the Lamé parameter $\mu$ by supposing a constant macroscopic Poisson ratio $v$, the oedometric bulk modulus is linked to the micro-porosity via the Biot coefficient as follows

$$
\tilde{M}^{b / t}=\frac{3(1-v)}{1+v} K_{s}\left(1-\alpha^{b / t}\right) .
$$

Water intake being very slow the system can be assumed at mechanical equilibrium instantaneously so that the total pressure of each part corresponds to the external swelling pressure. The bottom part being completely saturated $\mathrm{P}_{\mathrm{f}}^{\text {eff, }}=0$, whereas at the top part we have $P_{f}^{e f f, t} \approx-\left(1-\alpha^{t}\right) P_{c}^{i}=-\left(1-\alpha^{t}\right) \Pi_{d}^{i}$. The respective vertical components of the total pressure are written as

$-P_{e x t}=\sigma_{E}^{b}-\Pi_{\text {eff }}^{b}=\tilde{M}^{b} \varepsilon^{b}-\left(1-\alpha^{b}\right) \Pi_{d}^{b}$,

and

$-P_{\text {ext }}=-P_{f}^{e f f, t}+\sigma_{E}^{t}-\Pi_{e f f}^{t}=\tilde{M}^{t} \varepsilon^{t}+\left(1-\alpha^{t}\right)\left(\Pi_{d}^{i}-\Pi_{d}^{t}\right)$.

The disjoining pressure at the sufficiently hydrated bottom part is computed by PB law (6), whereas at the much drier top part it is given by the HS law in (7).

In a similar way, the respective micro-porosities are obtained from (9) which yields

$$
n_{f}^{b}=n_{f}^{i}+\frac{n_{f}^{b}\left(1-n_{f}^{b}\right)}{\frac{2\left(1-2 v_{s}\right)}{1+v_{s}}+n_{f}^{b}}\left(\varepsilon^{b}-\frac{\Pi_{d}^{b}}{K_{s}}\right),
$$

and

$$
n_{f}^{t}=n_{f}^{i}+\frac{n_{f}^{t}\left(1-n_{f}^{t}\right)}{\frac{2\left(1-2 v_{s}\right)}{1+v_{s}}+n_{f}^{t}}\left(\varepsilon^{t}+\frac{\Pi_{d}^{i}-\Pi_{d}^{t}}{K_{s}}\right) .
$$

The nano-porosities are expressed starting from (8) and read as

$$
\begin{aligned}
\left(1-n_{f}^{b}\right)\left(1-\phi^{b}\right) & =\left(1-n_{f}^{i}\right)\left(1-\phi^{i}\right) \exp \left(-\varepsilon^{b}\right) \\
& =\left(1-n_{f}^{i}\right)\left(1-\phi^{i}\right) \frac{x}{x+\Delta},
\end{aligned}
$$

and 


$$
\begin{aligned}
\left(1-n_{f}^{t}\right)\left(1-\phi^{t}\right) & =\left(1-n_{f}^{i}\right)\left(1-\phi^{i}\right) \exp \left(-\varepsilon^{t}\right) \\
& =\left(1-n_{f}^{i}\right)\left(1-\phi^{i}\right) \frac{L-x}{L-x-\Delta} .
\end{aligned}
$$

To derive the above expressions we adopted an approximation of the elementary deformation $\mathrm{d} \varepsilon$ as a function of the elementary variation of the sample height $\mathrm{dl}$ as follows $\mathrm{d} \varepsilon=\mathrm{dl} / 1$. Integration of this equation yields

$$
[\varepsilon]_{0}^{\varepsilon^{b}}=[\ln l]_{x}^{x+\Delta} \rightarrow \varepsilon^{b}=\ln \frac{x+\Delta}{x},
$$

and

$$
[\varepsilon]_{0}^{\varepsilon^{t}}=[\ln l]_{L-x}^{L-x-\Delta} \rightarrow \varepsilon^{t}=\ln \frac{L-x-\Delta}{L-x} .
$$

We obtain a system of 6 unknowns $\left\{\mathrm{P}_{\text {ext }} \mathrm{x}\right.$ (or $\Delta$ ), $\left.\mathrm{n}_{\mathrm{f}}^{\mathrm{b}, \mathrm{t}}, \phi^{\mathrm{b} / \mathrm{t}}\right\}$ depending on $\mathrm{S}_{\mathrm{w}}$ parameterized by $\mathrm{K}_{\mathrm{s}}, v$ and $v_{\mathrm{s}}$.

\section{Results and Discussion}

We now depict the numerical results of the simulation of the swelling pressure during hydration and the related evolution of the microscopic properties giving further insight to the local rearrangement. As the present study has a qualitative character, without any loss of generality we assume identical Poisson ratios at the macro- and micro-scale, $v_{\mathrm{s}}=v=0.25$. The numerical results are analyzed in terms of $\mathrm{K}_{\mathrm{s}}$ playing the role of a scaling parameter accounting for the transmissibility between nanoscopic disjoining and microscopic capillary effects.

\subsection{Swelling pressure}

Figure 5 shows the external swelling pressure for saturations between $\mathrm{S}_{\mathrm{w}}{ }^{\mathrm{i}}$ and $\mathrm{S}_{\mathrm{w}}{ }^{\mathrm{f}}=1$. The numerical results for different values of $\mathrm{K}_{\mathrm{s}}(1 \mathrm{MPa}, 5 \mathrm{MPa}$ and $100 \mathrm{MPa})$ are compared to the experimental values taken from [1].

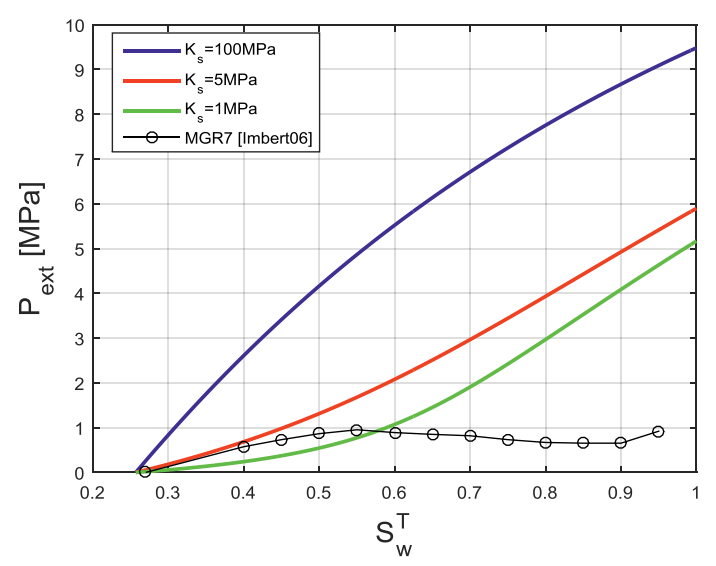

Figure 5. External swelling pressure vs. saturation parameterized by the microscopic bulk modulus and comparison with experimental data from [1].

In agreement with the experimental results, $P_{\text {ext }}$ increases during water intake. Owing to the separation of the different contributions (contact stress, capillary pressure and disjoining forces) to the total pressure (1), the model shows that the increase in the swelling pressure is not due to an increase of the disjoining pressure.
As shown on Figure 6 for $\mathrm{K}_{\mathrm{s}}=5 \mathrm{MPa}, \Pi_{\mathrm{d}}$ decreases during water infiltration. The red dots correspond to the top part of the sample being at initial saturation where $\Pi_{d}$ is computed by a HS law (7), whereas the blue dots correspond to the bottom part being at full saturation and where $\Pi_{d}$ is given by PB theory (6). As capillary pressure vanishes at full saturation and deformation is restricted, the external pressure is completely determined by the disjoining pressure which is given by $\mathrm{PB}$ law as the overall medium is now composed of the bottom part. In agreement with the expectations [18], the completely saturated state corresponds to about 3 water layers in the nanopores.

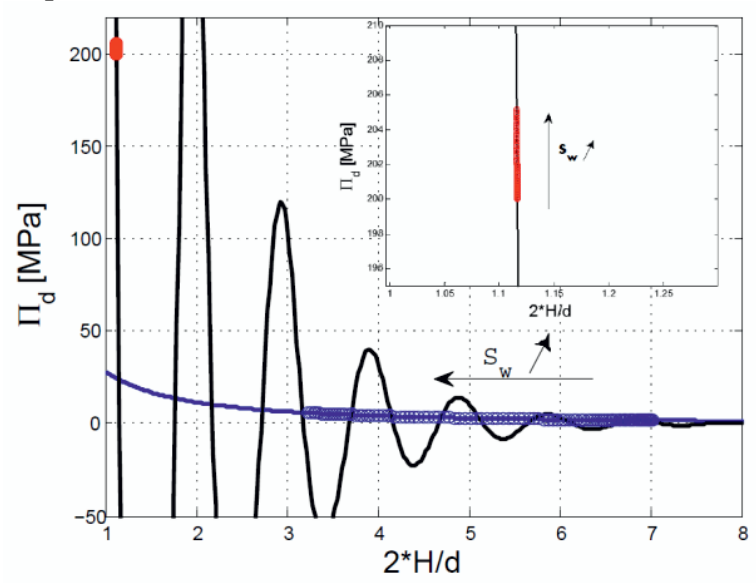

Figure 6. Evolution of the disjoining pressure with saturation: in the top dry part (red dots) computed by a HS law (7) and in the bottom part (blue dots) by $\mathrm{PB}$ theory (6); $\mathrm{K}_{\mathrm{s}}=5 \mathrm{MPa}$.

Figure 5 highlights also the limits of the present model. The best fit to the experimental data is observed for $\mathrm{K}_{\mathrm{s}}=5 \mathrm{MPa}$. After a good agreement at low saturations, the numerical values remain clearly superior to the experimental ones. This discrepancy is even more pronounced for higher values of $\mathrm{K}_{\mathrm{s}}$. Setting $\mathrm{K}_{\mathrm{s}}$ to $1 \mathrm{MPa}$ allows reducing the swelling pressure at full saturation, but the evolution of $\mathrm{P}_{\mathrm{ext}}$ during hydration does not enhance the fit to the experimental observations. Notwithstanding these shortcomings it can be concluded that in order to obtain swelling pressures in the $\mathrm{MPa}$ range, $K_{\mathrm{s}}$ has to be of the same order of magnitude which is clearly inferior to the global values of the bulk modulus given in the literature and varying between $10-50 \mathrm{GPa}$ $[21,22]$. Since $\mathrm{K}_{\mathrm{s}}$ being solely linked to the connectivity of the solid structure inside the clay agglomerates, we conclude from this observation that the overall rigidity may be ensured by the action of the disjoining forces originating from the nanoscopic clay platelet scale.

It is noteworthy that, conversely to the numerical data, after in initial increase the experimental swelling pressure decreases to increase again. Such behaviour stems from the collapse of the macrostructure when reaching the LC line in the frame of elasto-plastic deformation [2] which is not accounted for in the present model approach. This multi-scale model allows to give insight to the contribution of local phenomena to the macroscopic mechanical behaviour. As a next step one has to incorporate macroscopic plastic deformation. 


\subsection{Porosities at the fully saturated state}

Figure 7 shows the nano- and the microporosities at the fully saturated state as a function of the microscopic bulk modulus. In agreement with the expectations, the microporosity decreases between the initial dry $\left(\mathrm{n}_{\mathrm{f}}^{\mathrm{i}}=0.39\right)$ and the final saturated state, which is linked to capillary pressure decrease $[3,6]$. The volume being fixed impeding any macroscopic deformation, the nanoporosity increases during water intake. We remind that initially $\phi i=0.24$. This confirms the ability of the multi-scale model in capturing water transfer from the microscopic inter particle to the nanoscopic inter platelet pores during hydration. Such change in the porous space is more pronounced for a soft solid matrix being characterized by low values of $\mathrm{K}_{\mathrm{s}}$. Note finally that the increase of the inter platelet distance is at the origin of the decrease of the disjoining pressure during hydration.

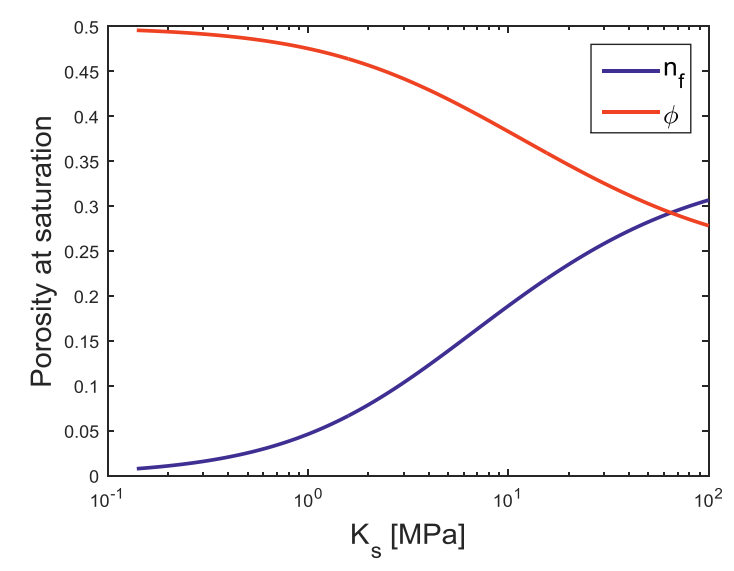

Figure 7. Nano- and microporosities at the fully saturated state as a function of the microscopic bulk modulus $\mathrm{K}_{\mathrm{s}}$.

\section{Conclusion}

Our former developed multi-scale version of Terzaghi's effective stress principle for unsaturated expansive clays accounting for electro-chemico-hydro-mechanical coupling at different length-scales is applied to numerically simulate swelling pressure tests at constant volume. The comparison between our numerical results and experimental data performed at the CIEMAT highlight the ability of our model in capturing the contribution of microscopic capillary pressure and nanoscopic disjoining forces to the overall swelling pressure. The main conclusions are:

- The model is able to capture water transfer from microscopic inter particle to nanoscopic inter platelet pores during hydration. As a consequence the increase of the swelling pressure is the result of two phenomena: Initially the high capillary pressure is completely compensated by the disjoining pressure. At full saturation where capillary pressure vanishes the swelling pressure corresponds to the effective disjoining pressure with a somewhat lower magnitude due to a higher inter platelet distance within the clay agglomerates at the nano-scale. - In spite of classical PB theory remaining valid at high saturation, the molecular character of the electrolyte solution has to be taken into account for accurately modelling the disjoining pressure at small inter-platelet distances. By selecting a nanoscopic description of a simple HS fluid confined between two planar hard walls the disjoining pressure has been computed using classical DFT. Notwithstanding the simplicity of our approach it leads to an improvement of the nanoscopic description particularly at low hydration.

- The microscopic bulk modulus plays the role of a scaling parameter between disjoining and capillary effects. The numerical results show that in order to obtain swelling pressures in the $\mathrm{MPa}$ regime, $\mathrm{K}_{\mathrm{s}}$ has to be of the same order of magnitude, which is significantly lower than the values of the overall bulk modulus typically found in the literature. The overall rigidity of the medium may thus be ensured by nanoscopic disjoining forces.

Some shortcomings still persist such as the overestimation of the swelling pressure and the loss of accuracy in reproducing the decrease of the swelling pressure during water intake due to a rearrangement of the clay clusters. Such behaviour is tied up to a collapse of the macrostructure when reaching the LC line. Further work is ongoing to include plastic deformation.

\section{References}

1. C. Imbert, M.V. Villar, Appl. Clay Sci. 32, 197 (2006)

2. A. Gens, B. Valleján, M. Sánchez, C. Imbert, M.V. Villar, M.Van Geet, Géotechnique 61(5), 367 (2011)

3. A. Gens, E.E. Alonso, Can. Geotech. J. 29, 1013 (1992)

4. E.E. Alonso, J. Vaunat, A. Gens, Eng. Geol. 54, 173 (1999)

5. M. Sánchez, A. Gens, L. do Nascimento Guimarães, S. Olivella, Int. J. Numer. Anal. Meth. Geomech. 29, 751 (2005)

6. I.J.A. Brackeley, Int. Conf. Expansive Soils 1, 169 (1973)

7. H. Komine, N. Ogata, Can. Geotech. J. 31, 478 (1994)

8. C. Moyne, M.A. Murad, Transport Porous Med. 62, 333 (2006)

9. J. Mainka, M.A. Murad, C. Moyne, S.A. Lima, Vadose Zone J. 13(5) (2014)

10. E.E. Alonso, A. Gens, A. Josa, Géotechnique 40(3), 405 (1990)

11. A. Lloret, M.V. Villar, M. Sánchez, A. Gens, X. Pintado, E.E. Alonso, Géotechnique 53(1), 27 (2003)

12. J.-L. Auriault, C. Boutin, C. Geindreau, Homogenization of Coupled Phenomena in Heterogeneous Media (John Wiley \& Sons, Hoboken, NJ, 2009)

13. J.-M. Pereira, H. Wong, P. Dubujet, P. Dangla, Int. J. Numer. Anal. Meth. Geomech. 29(11), 1127 (2005)

14. P.F. Low, Langmuir 3, 18 (1987)

15. A. Olesky, J.-P. Hansen, J. Chem. Phys. 132, 204702 (2010)

16. R. Roth, J. Phys.: Condens. Matter 22, 063102 (2010) 
17. J.-P. Hansen, I.R. McDonald, Theory of Simple Liquids (Elsevier Science, $3^{\text {rd }}$ edition, 2006)

18. E. Ferrage, B. Lanson, L.J. Michot, J.-L. Robert, J. Phys. Chem. C 114, 4515 (2010)

19. R. T. Cygan, J-J. Liang, A.G. Kalinichev, J. Phys. Chem. B 108, 1255 (2004)

20. A.-M. Tang, Applied Unsaturated Soil Mechanics, MUSE School, Paris, May (2006)

21. B.E. Hornby, L.M. Schwartz, J.A. Hudson, Geophysics 59(10), 1570 (1994)

22. C.M. Sayers, Geophys. Prospect. 61, 416 (2013) 